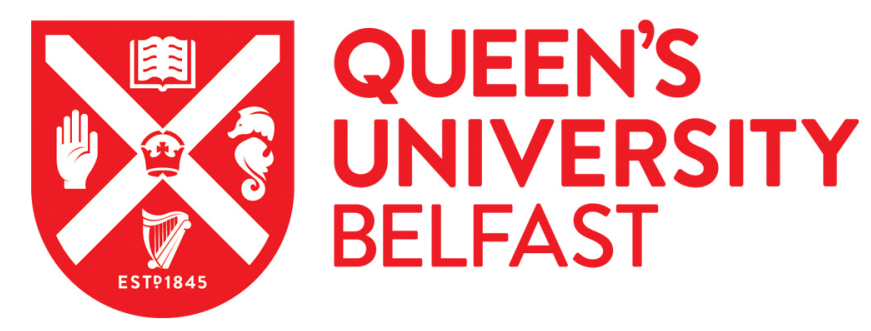

\title{
A Computational Evaluation of the Diffusion Mechanisms for C8 Aromatics in Porous Organic Cages
}

Jackson, E., Miklitz, M., Song, Q., Tribello, G., \& Jelfs, K. (2019). A Computational Evaluation of the Diffusion Mechanisms for C8 Aromatics in Porous Organic Cages. The Journal of Physical Chemistry C.

https://doi.org/10.1021/acs.jpcc.9b05953

\section{Published in:}

The Journal of Physical Chemistry C

Document Version:

Peer reviewed version

Queen's University Belfast - Research Portal:

Link to publication record in Queen's University Belfast Research Portal

Publisher rights

Copyright 2019 American Chemical Society. This work is made available online in accordance with the publisher's policies. Please refer to any applicable terms of use of the publisher.

\section{General rights}

Copyright for the publications made accessible via the Queen's University Belfast Research Portal is retained by the author(s) and / or other copyright owners and it is a condition of accessing these publications that users recognise and abide by the legal requirements associated with these rights.

Take down policy

The Research Portal is Queen's institutional repository that provides access to Queen's research output. Every effort has been made to ensure that content in the Research Portal does not infringe any person's rights, or applicable UK laws. If you discover content in the Research Portal that you believe breaches copyright or violates any law, please contact openaccess@qub.ac.uk. 


\section{A Computational Evaluation of the Diffusion}

\section{Mechanisms for C8 Aromatics in Porous Organic}

\section{Cages}

Edward Jackson, ${ }^{\dagger}$ Marcin Miklitz, ${ }^{\dagger}$ Qilei Song, ${ }^{\ddagger}$ Gareth A. Tribello, ${ }^{\llbracket}$ and Kim E. Jelfs $s^{* \dagger}$

$\dagger$ Department of Chemistry, Imperial College London, Molecular Sciences Research Hub, White City Campus, Wood Lane, London W12 0BZ, United Kingdom $\ddagger$ Department of Chemical Engineering, Imperial College London, South Kensington Campus, London, SW7 2AZ, United Kingdom

ФAtomistic Simulation Centre, School of Mathematics and Physics, Queen's University Belfast, University Road, Belfast BT9 1NN, United Kingdom

E-mail: k.jelfs@imperial.ac.uk 


\begin{abstract}
The development of adsorption and membrane-based separation technologies toward more energy and cost efficient processes is a significant engineering problem facing the world today. An example of a process in need of improvement is the separation of $\mathrm{C} 8$ aromatics to recover para-xylene, which is the precursor to the widely used monomer terephthalic acid. Molecular simulations were used to investigate whether the separation of C8 aromatics can be carried out by the porous organic cages CC3 and CC13, both of which have been previously used in the fabrication of amorphous thin film membranes. Metadynamics simulations showed significant differences in the energetic barriers to the diffusion of different C8 aromatics through the porous cages, especially for CC3. These differences imply that meta-xylene and ortho-xylene will take significantly longer to enter or leave the cages. Therefore, it may be possible to use membranes composed of these materials to separate ortho- and meta-xylene from para-xylene by size exclusion. Differences in the C8 aromatics' diffusion barriers were caused by their different diffusion mechanisms, while the lower selectivity of CC13 was largely down to its more significant pore breathing. These observations will aid the future design of adsorbents and membrane systems with improved separation performance.
\end{abstract}

\title{
Introduction
}

Molecular separation processes are estimated to be responsible for 10-15\% of the world's total energy consumption. ${ }^{1,2}$ Therefore, one of the key economic and environmental challenges today is the development of less energy-intensive alternatives to commonly used separation processes such as distillation. The separation of the aromatic molecule para-xylene (pX) from its structural isomers meta-xylene $(\mathrm{mX})$, ortho-xylene $(\mathrm{oX})$ and ethylbenzene (Figure 1 ) is an example of a separation in need of an alternative process and was named one of "seven separations to change the world" by Sholl and Lively. ${ }^{2}$ Millions of tonnes of pX 
are used each year in the production of many polyester fibres and the production of the polymer polyethyleneterephthalate(PET), ${ }^{3}$ thus it is a precursor of considerable industrial importance. ${ }^{3,4}$

The xylene structural isomers share similar physical properties that complicate pX separation; for instance, nearly-identical boiling points reduce the effectiveness of distillation. ${ }^{5}$ The most common isomer separation method for $\mathrm{pX}$ involves a simulated moving bed (SMB) containing a zeolite which preferentially adsorbs $\mathrm{pX}{ }^{6,7}$ The Parex process that was developed by UOP is an example of an SMB process. ${ }^{8}$ A downside of SMB processes is that they require the use of a competitive desorbent to remove $\mathrm{pX}$ from the zeolite bed; a further separation, usually using distillation, is then required to remove the desorbent from the pX product. ${ }^{9}$ The different freezing points of the four isomers enable separation by crystallisation; however, the method is highly energy intensive. ${ }^{5}$
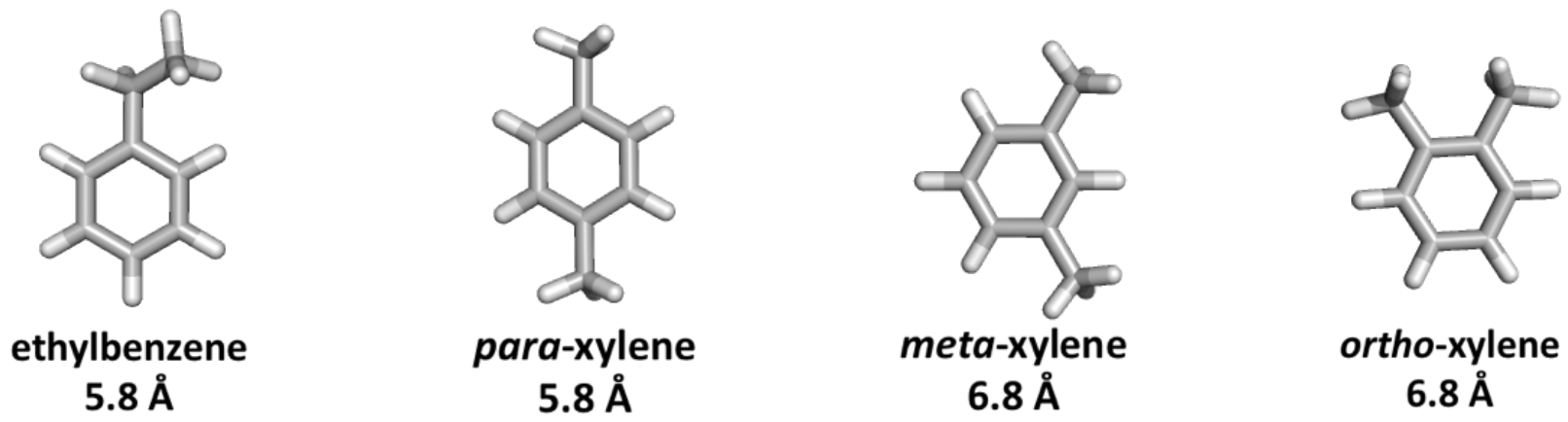

Figure 1: Ethylbenzene, the three xylene isomers, and their kinetic diameters. ${ }^{10}$

There is a significant precedent for the use of porous materials in the separation of C8 aromatics, as shown by the widespread application of zeolites in the Parex process. ${ }^{6}$ Zeolites are also used as catalysts in xylene isomerization reactions, which are used in tandem with SMB or crystallization separations to increase the overall pX yield. ${ }^{7,11}$ Increasingly, metalorganic frameworks (MOFs) are also being investigated for this separation. For adsorptionbased separations, Torres-Knoop et al. found that MAF-X8, a Zn pyrazolate-carboxylate MOF, was selective for $\mathrm{pX}$ due to the presence of channels which allowed $\pi$-stacking of the pX molecules. ${ }^{12}$ Gee et al. also reported several MOFs with a pX selectivity comparable 
to the BaX zeolite that is used in the Parex process. ${ }^{13}$ Both MIL- $47^{13}$ and UiO- $66^{14}$ have been reported to be selective for oX, and a separation using one of these two MOFs can compliment existing pX-selective methods.

Shape-selective membranes provide an alternative for the separation of xylene isomers, which Sholl and Lively claim would use $90 \%$ less energy than traditional methods. ${ }^{2}$ Some state-of-the-art membrane materials under investigation include zeolites and MOFs. Both zeolites and MOFs contain well-defined interconnected channels with nanometer-sized apertures that allow pX (kinetic diameter $=5.8 \AA$ ) to be separated from the bulkier $\mathrm{mX}$ and oX (kinetic diameter $=6.8 \AA$ ). MFI zeolite membranes in particular have been extensively studied for pX production. ${ }^{6,15-18}$ The manufacture of crystalline zeolites and MOFs into large-scale and defect-free membranes remains challenging, however. Solution-processable polymers of intrinsic microporosity (PIMs) are a promising alternative for chemical separations. Polymeric membranes tend to present a broad size distribution of micropores and swell in the presence of organic chemicals leading to poor separation selectivity. ${ }^{19}$ Carbon molecular sieve (CMS) membranes have demonstrated great potential for the separation of xylene isomers due to their ordered pore architectures, high diffusion selectivity, and ease of manufacture. ${ }^{20}$

Porous organic cages are a class of porous materials that are made of discrete molecules with intrinsic voids, which can self-assemble to form extended pore networks in the solid state. ${ }^{21-23}$ These materials have many potential uses in the field of separation, including in the sensing and removal of the pollutant formaldehyde from air; ${ }^{24}$ the removal of the greenhouse gas $\mathrm{SF}_{6}$ from air; ${ }^{25}$ the separation of noble gases; ${ }^{26}$ and the separation of aromatic molecules,${ }^{27}$ including enantiomers of chiral aromatics. ${ }^{26}$ One key advantage of porous molecular materials over extended framework materials, such as MOFs or zeolites, is their high solution processability, which comes from their ability to dissolve in solvents without disassembling. ${ }^{28}$ Both amorphous ${ }^{29}$ and crystalline ${ }^{30}$ porous organic cage thin films can be fabricated by solution casting from organic solvents such as dichloromethane. The resultant 
amorphous thin film membranes are capable of a shape-selective separation of $\mathrm{H}_{2}$ and $\mathrm{N}_{2} \cdot{ }^{29}$ This promising membrane separation performance suggests that porous organic cages could potentially be used in shape-selective membranes for the separation of aromatic molecules. This work focuses on computational evaluations of the diffusion of $\mathrm{C} 8$ aromatics in two imine-based porous organic cages (Figure 2): CC3, which is constructed from triformylbenzene building blocks connected by cyclohexane diamine linkers, ${ }^{31,32}$ and CC13, which uses the same building block but with 1,1-dimethyl diamine. ${ }^{33}$

(a)

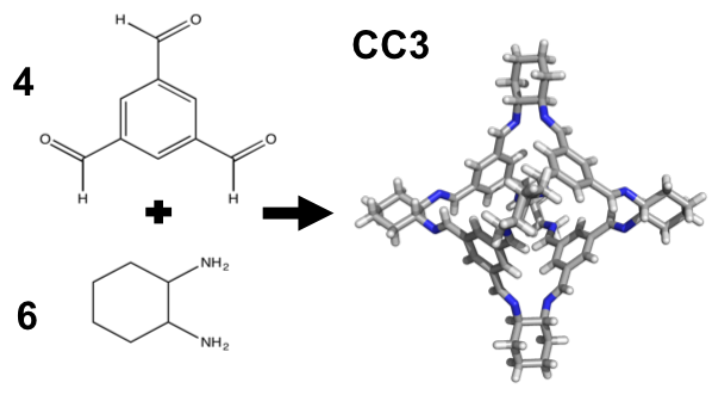

(b)

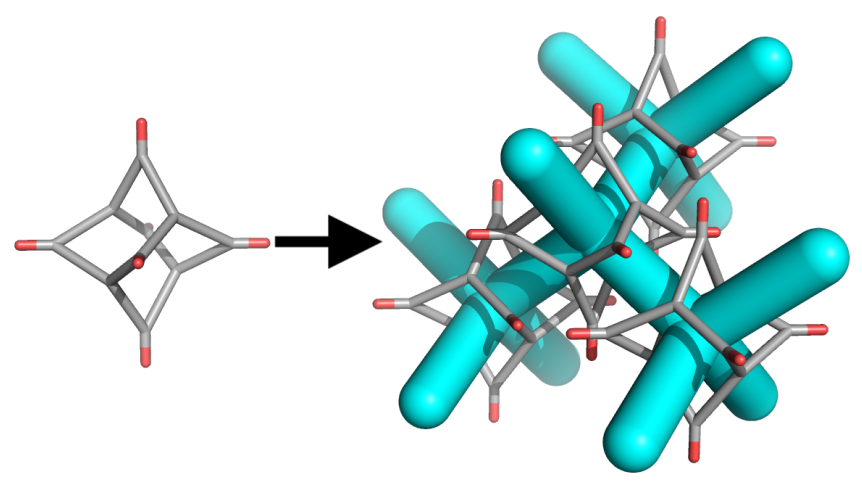

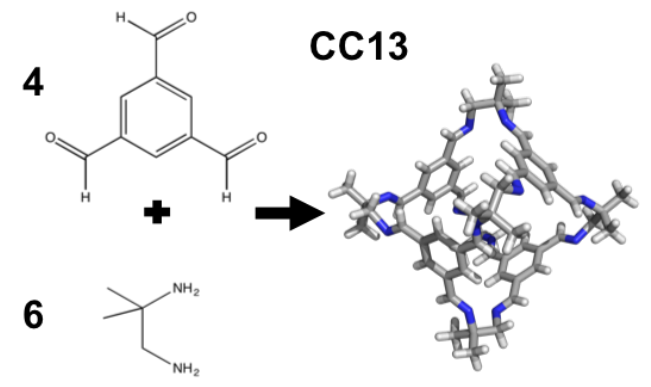

(c)

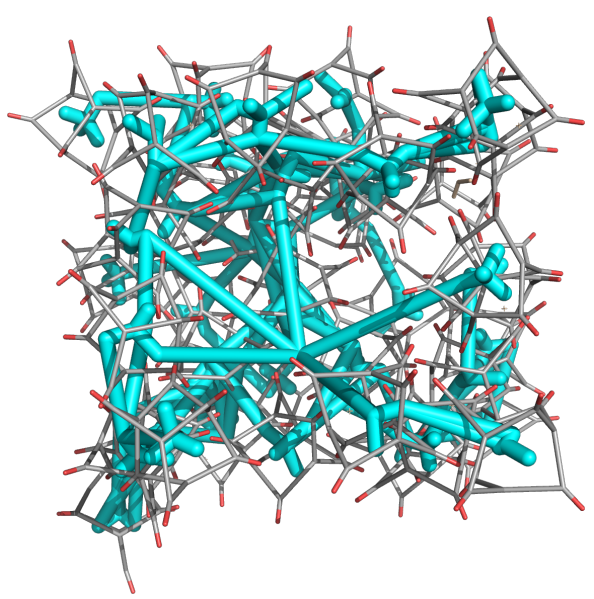

Figure 2: a) The porous organic cages CC3 and CC13 and their precursors. The molecules differ only in the vertex functionalization. b) A simplified wireframe CC3 molecule (gray $=$ aromatic building block, red $=$ cyclohexane linker), and the crystal structure and pore network of its $\alpha$-polymorph (cyan $=$ pore network). c) A wireframe model of an amorphous CC3 structure previously reported in the literature. ${ }^{34}$

CC3 and CC13 have several properties that would make them ideal for the shapeselective separation of $\mathrm{C} 8$ aromatics, including the diameters and flexibility of the cage 
windows. The static pore limiting diameter of a single CC3 cage is $3.62 \AA,{ }^{35}$ which is far too small to allow the C8 aromatic molecules in Figure 1 to diffuse through. However, the windows in porous organic cages, including CC3 and CC13, are capable of dynamic changes in size. In other words, the cages can expand to accommodate large guests ${ }^{25,26,35,36}$ or provide access to formally occluded voids in a crystal structure. ${ }^{37,38}$ This phenomenon is similar to Barbour's "porosity without pores" 39 and Holden et al. defined three classes of porosity as a result of this observation. The first class is static porosity, which is porosity that is present even in a static crystal structure. The second is dynamic porosity, which is not present in the static crystal structure but instead results from the dynamic motion of the cage molecules. The third and final type is cooperative porosity: porosity which can only exist due to the presence of a guest molecule in the pore network. ${ }^{38}$ Diffusion mechanisms that take advantage of cooperative porosity have been observed for many guest molecules that, under normal circumstances, are too large to pass through a host's pore network. Examples of this include the diffusion of linear alkanes and $\mathrm{SF}_{6}$ in ZIF-8, ${ }^{40}$ the diffusion of $\mathrm{SF}_{6}$ in $\mathbf{C C} 3,{ }^{25}$ and the diffusion of xylene isomers in UiO-66. ${ }^{14}$

Consideration of dynamic and cooperative porosity is essential to gain an accurate picture of the sorption behavior of a porous organic cage, especially when considering guests whose diameters are similar to or larger than the limiting diameter of the cage windows. Diffusion of guest molecules can be explored using molecular dynamics (MD) in many cases. Values for a guest's self-diffusivity in a porous network can be calculated once ballistic motion through the pore network has been achieved, which, for smaller guests, can require simulation times of less than $10 \mathrm{~ns}$ at $298 \mathrm{~K} .{ }^{36}$ High-temperature MD has also been used to observe the diffusion of large guests, such as xylene isomers in CC3 ${ }^{27}$ Alternatively, enhanced sampling methods can be used to understand the cooperative diffusion of large guest molecules. ${ }^{41-44}$ Umbrella sampling is one example of an enhanced sampling method, which uses artificial harmonic restraint forces to limit the motion of a guest molecule in a system. ${ }^{45}$ Camp and Sholl used umbrella sampling to identify the cooperative diffusion mechanisms for large guest 
molecules such as $\mathrm{SF}_{6}$ in the $\mathbf{C C} 3$ crystal structure and gave a quantitative estimate for the diffusivity of $\mathrm{SF}_{6}$ in $\mathbf{C C} 3{ }^{43}$ By contrast, no diffusion of $\mathrm{SF}_{6}$ could be measured in a $10 \mathrm{~ns}$ MD simulation. ${ }^{36}$

An alternative enhanced sampling method is the metadynamics method of Laio and Parrinello. ${ }^{46}$ Metadynamics has been used to observe the diffusion of $\mathrm{SF}_{6}$ in $\mathbf{C C} 3$ and to determine the diffusion mechanism and associated energetic barrier. ${ }^{25}$ It has also been used to determine the energy barriers to Xe and Kr diffusion in CC3 and other porous organic cages and cage-like molecules in a search for new noble gas separating agents. ${ }^{47}$ In this work, metadynamics is used to evaluate key energetic barriers to the diffusion of $\mathrm{C} 8$ aromatics in the porous organic cages $\mathbf{C C} 3$ and $\mathbf{C C} 13$.

\section{Methods}

\section{Validation of the Single Molecule Approach}

In this study, calculations were carried out on isolated CC3 and CC13 single molecular cages, as opposed to the cages' solid-state crystal structures. Calculations with single cages have been previously used to evaluate barriers to the diffusion of $\mathrm{SF}_{6},{ }^{25} \mathrm{Xe}$ and $\mathrm{Kr}^{47}$ in CC3. The main advantage of studying single cages compared to solid-state structures is that it allows for a faster exploration of properties intrinsic to a particular cage that may affect its ability to separate a set of molecules. Furthermore, Miklitz et al. showed that window size fluctuations, which are crucial to a cage's ability to separate large guests, show mostly the same distribution in an isolated CC3 cage and the crystal structure of its $\alpha$ polymorph. ${ }^{47}$ The single molecule approximation also allows much longer timescales, such as the 100 ns previously required to evaluate the energetic barrier to $\mathrm{SF}_{6}$ diffusion ${ }^{25}$ and the even longer timescales applied in this work, to be accessed at a reasonable computational cost. Some properties can vary based on the cage's packing and therefore cannot be determined using the single molecule approximation. For example, the preferred binding site of $\mathrm{pX}$ in 
the CC3 crystal structure is in the inter-cage void, ${ }^{27}$ which is not present in a single cage model. The self-diffusivity of a particular guest molecule also cannot be calculated using this approximation because a guest molecule's diffusion behavior can vary between crystalline and amorphous structures of the same cage. ${ }^{34,36}$ However, several significant energetic barriers can be calculated independently of the bulk structure. These barriers are the barrier to crossing through the cage windows and the barrier to the reorientation of a guest molecule in the cage so that it can leave through a different window. An understanding of this reorientation barrier is necessary because the molecules must reorient themselves in order to move through the tetrahedral pore network of CC3 $\alpha .{ }^{27}$ The motions leading to these barriers are shown schematically in Figure 3a. The differences in the window and reorientation barriers for each aromatic guest will give a qualitative view into whether the separation is viable using a particular cage, which would provide an efficient route for screening porous organic cage systems for separation applications.
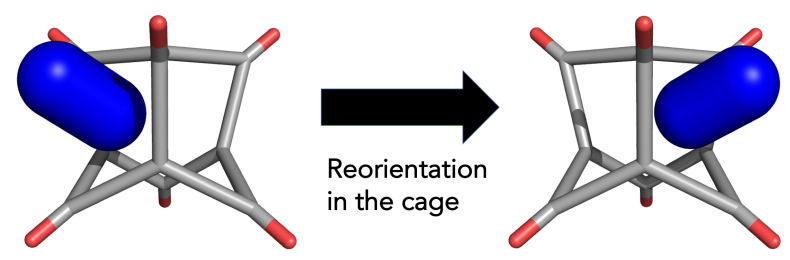

(a)

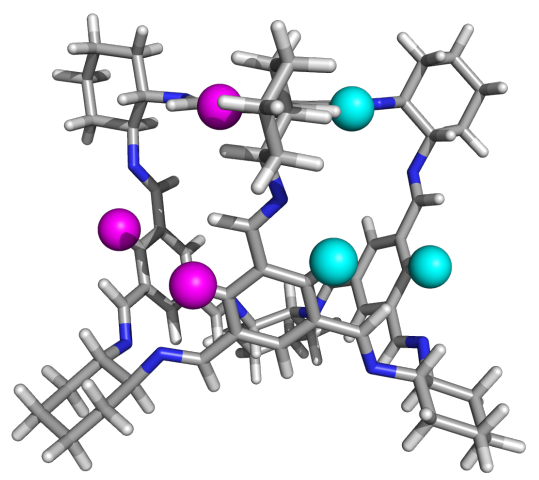

(b)

Figure 3: a) A schematic showing a generic guest molecule (blue cylinder) entering a schematic molecular cage (gray = aromatic building block, red = cyclohexane linker) by one window and exiting by another. There are potentially three distinct energetic barriers to this diffusion process; passing through the first window; reorienting in the cage so as to be able to exit through a different window and finally passing through the second window in order to leave the cage. b) The windows used to define the collective variable for the metadynamics simulations, with the hydrogen atoms used to approximate the window centers highlighted in magenta (window 1) and cyan (window 2). 


\section{Calculation of Free Energy Barriers to Diffusion}

The single cage molecular structures for CC3 and CC13 were extracted directly from the Cambridge Structural Database (CSD), reference codes PUDXES ${ }^{32}$ and OFOQEI, ${ }^{33}$ respectively. For the CC13 molecule, a single isomer of the cage was generated by resolving the positions of the linker atoms with a Python script (see Section S1 for details). Single molecules of $\mathrm{pX}, \mathrm{mX}, \mathrm{oX}$, ethylbenzene, benzene, and toluene were manually inserted into each of the cages and each structure was used as an input for a simulation using DL_POLY2.20, ${ }^{48}$ with the input structures generated using DL_FIELD3.5..$^{49,50}$ The OPLS_2005 force field ${ }^{51}$ was chosen for the simulations, as the OPLS family of force fields has proven effective at modeling imine-based porous organic cages, ${ }^{52}$ including the calculation of diffusion barriers ${ }^{25}$ and pathways. ${ }^{37}$

The metadynamics calculations were carried out using DL_POLY2.20 and the PLUMED 2 plugin. ${ }^{53}$ Two collective variables were used: the distances between the centers of two of the cage's windows and the center of mass of the guest molecule. These collective variables were designed to encompass all three energetic barriers described in Figure 3a. Furthermore, energetic walls were used to prevent the guest molecule from moving more than $4.5 \AA$ from the cage center of mass and from entering the other two cage windows. Window centers were approximated by computing the center of mass of the three aromatic hydrogen atoms surrounding each window of the cage. The particular hydrogen atoms that were used in the collective variables and that were employed in the metadynamics simulations are shown in Figure 3b. Two-dimensional Free Energy Surfaces (FESs) were generated using the sum_hills utility of PLUMED and the lowest energy pathways across the FESs were found using the MEPSA code developed by Marcos-Alcade et al. ${ }^{54}$ The lowest energy pathways across the FES encompassed two window crossings and a reorientation. We also computed these FESs using a reweighting procedure. ${ }^{55,56}$ Error bars on these estimates of the free energy were estimated using a block averaging procedure ${ }^{57}$ and were found to be on the order of $1 \mathrm{~kJ} \mathrm{~mol}^{-1}$ to $2 \mathrm{~kJ} \mathrm{~mol}^{-1}$. Finally, snapshots of the trajectories were visualized using the VMD package ${ }^{58}$ 
to explore the guest diffusion mechanisms. Full simulation details and an example input file for these metadynamics simulations can be found in Section S2.1, while the convergence of the simulations is discussed in Section S3.

\section{Calculation of Pore Limiting Envelopes}

A pore limiting envelope (PLE) is a histogram of the window sizes of a particular cage over time. The PLEs for CC3 and CC13 with no guest and the PLEs with single molecules of ethylbenzene, pX, mX and oX were calculated using an approach similar to one reported previously. ${ }^{47}$ MD simulations in the NVT ensemble were run using DL_POLY2.20 for each combination of guest and cage. The simulations used a timestep of $0.7 \mathrm{fs}$ and were run for $105 \mathrm{~ns}$ at $300 \mathrm{~K}$ using a Nose-Hoover thermostat. Atomic positions were sampled every $1.4 \mathrm{ps}$, resulting in 75,000 total configurations that were then analyzed using pywindow, a

Python package developed to calculate the structural properties of porous materials. ${ }^{59}$ For each cage, the final PLE histogram was generated from the diameters of all sampled windows (4 per cage over each of the sampled configurations).

For the simulations with a guest molecule present, the decision was made to not apply an energetic wall near the cage. This is different to the metadynamics simulations described in the previous section, in which the wall was used artificially encourage guest molecules to remain close to the window. The second set of metadynamics simulations were run using the same collective variable as the one used by Hasell et al. to study the diffusion of $\mathrm{SF}_{6}$ in CC3 and the changes in window size during this process. ${ }^{25}$ This collective variable samples the distance between the center of mass of the cage and the center of mass of the aromatic guest, with an energetic wall placed $24 \AA$ away from the cage center of mass to prevent the guest from moving too far from the cage (see Section S2.2 for further details and an example input file). PLEs were constructed from these metadynamics simulations by selecting only those sampled configurations where at least part of the guest molecule was in the window, defined as a collective variable value in the range of $2 \AA$ to $6 \AA$, and then only including the 
largest of the four window diameters in that configuration (which was assumed to be the one containing the guest).

\section{Results and Discussion}

We will first discuss the diffusion pathways and the associated free energy barriers for the six guest molecules passing through CC3, before comparing to these properties in CC13 to determine the influence of vertex functionalisation.

\section{Diffusion Free Energy Barriers in CC3}

We first explored the diffusion pathway for the six guest molecules (the four $\mathrm{C} 8$ aromatics in Figure 1, benzene and toluene) in CC3. The 2D FESs are shown in Figure 4, which also includes an annotated FES plot (Figure 4a) to assist in the interpretation of the results for each guest molecule. Figure 4c shows the 2D FES plots for each guest molecule in CC3. The minimum energy paths are represented in Figure 4c by a dotted line that indicates the approximate trajectory, ignoring small local energy fluctuations. It is important to not place too great a significance on small energy differences (less than a few $\mathrm{kJ} \mathrm{mol}^{-1}$ ) between barriers calculated with metadynamics simulations using a forcefield, due to the combination of potential errors with the forcefield and the limitations of the convergence criteria discussed in Section S3. The minimum energy paths in this two-dimensional CV space that were obtained by applying MEPSA ${ }^{54}$ on these free energy surfaces are shown in Figure S16a. The energy profiles for the minimum energy paths are shown in Figure $4 \mathrm{~b}$ and the values for the key energetic barriers and calculated errors in the reweighted FESs are shown in Table 1.

The top left plot in Figure 4c shows the FES plot for benzene diffusion through CC3, where the minimum energy path for diffusion passes close to the cage center of mass. Benzene passing close to the center of mass gives the guest/host complex shown in the first image in 
(a)

(b)
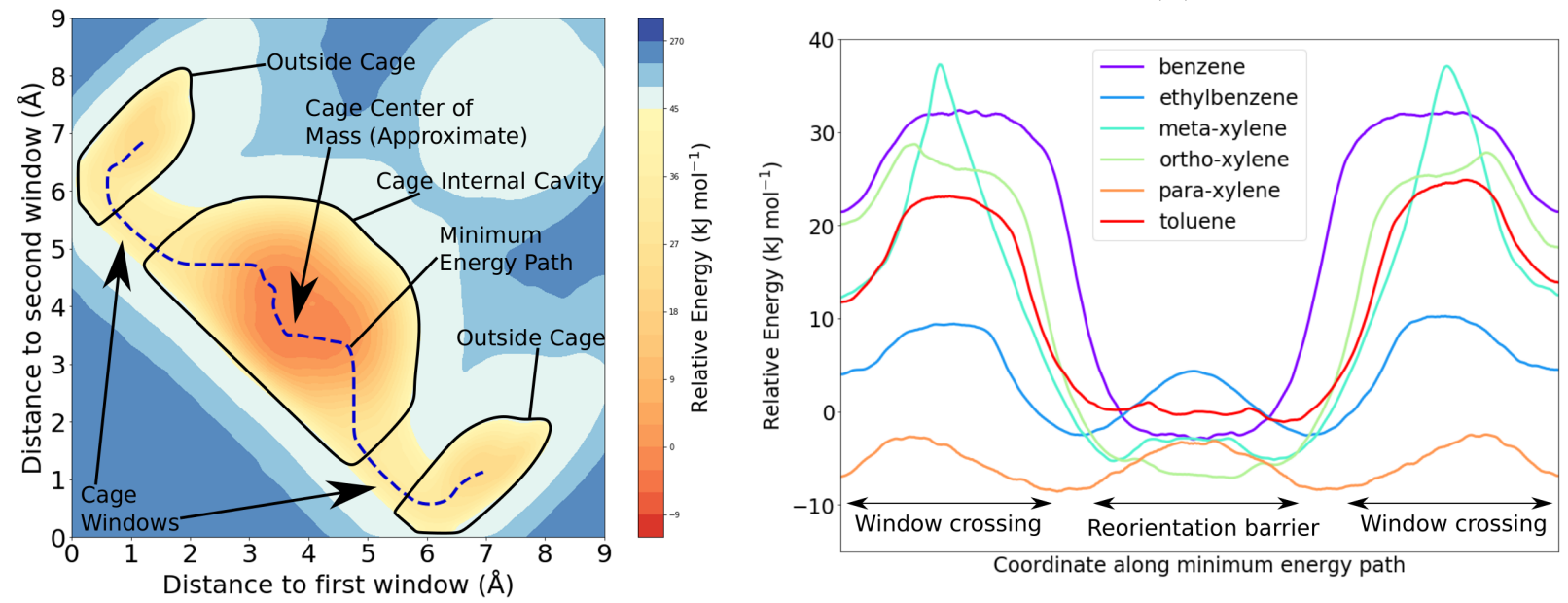

(c)
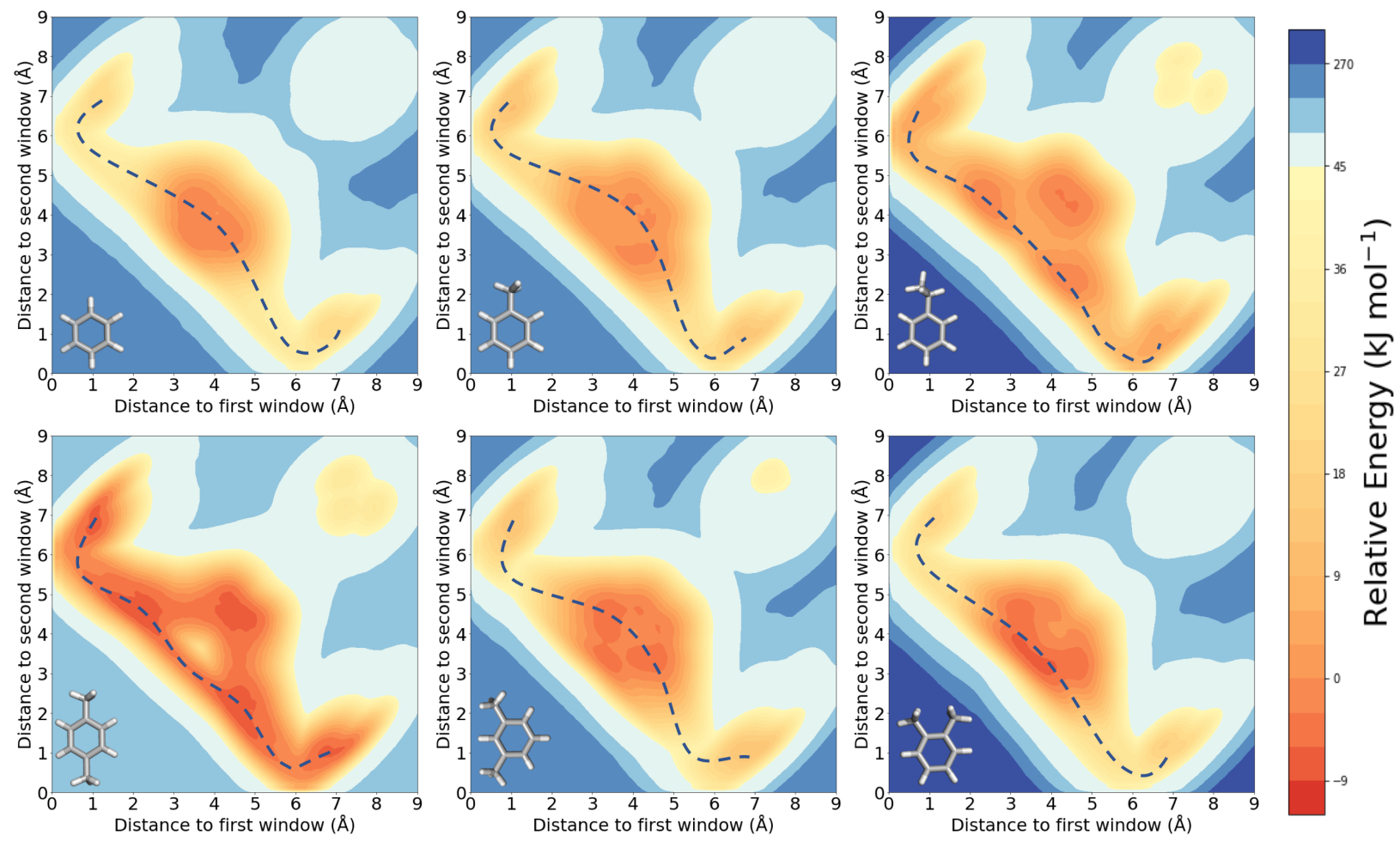

Figure 4: a) An annotated 2D FES, highlighting the key features of the plot, and their relation to features of the actual cage, and the minimum energy pathway that the guest will travel along as it moves between the two cage windows. Energies are relative to the point $(4,4)$, which is approximately in the center of the cage. For a further discussion of the plot's features, see Section S4. b) The free energy profile for the minimum energy path for each aromatic guest crossing through a CC3 cage. c) The 2D FES for each guest within CC3, including an approximate minimum energy path (dotted line). The guest is shown in the bottom left-hand corner of each plot. 
Table 1: The calculated window crossing and reorientation barriers for aromatic guest molecules in CC3 and CC13. The largest energetic barrier(s) for each guest/cage pair are highlighted in bold.

\begin{tabular}{|c|c|c|c|c|c|c|}
\hline Guest & $\begin{array}{l}\text { Window } \\
\text { Barrier } \\
\left(\mathrm{kJ} \mathrm{mol}^{-1}\right)\end{array}$ & \begin{tabular}{l}
\multicolumn{1}{c}{ CC3 } \\
Reorientation \\
Barrier \\
$\left(\mathrm{kJ} \mathrm{mol}^{-1}\right)$
\end{tabular} & $\begin{array}{l}\text { FES } \\
\text { Error } \\
\left(\mathrm{kJ} \mathrm{mol}^{-1}\right)\end{array}$ & $\begin{array}{l}\text { Window } \\
\text { Barrier } \\
\left(\mathrm{kJ} \mathrm{mol}^{-1}\right)\end{array}$ & $\begin{array}{l}\quad \text { CC13 } \\
\text { Reorientation } \\
\text { Barrier } \\
\left(\mathrm{kJ} \mathrm{mol}^{-1}\right)\end{array}$ & $\begin{array}{l}\text { FES } \\
\text { Error } \\
\left(\mathrm{kJ} \mathrm{mol}^{-1}\right)\end{array}$ \\
\hline $\mathrm{mX}$ & 43 & 2 & 2.2 & 34 & 0 & 1.5 \\
\hline oX & 35 & 2 & 1.9 & 31 & 0 & 1.6 \\
\hline benzene & 35 & 1 & 2.3 & 29 & 0 & 2.2 \\
\hline toluene & 25 & 1 & 2.1 & 27 & 0 & 1.6 \\
\hline ethylbenzene & 13 & 7 & 1.6 & 8 & 3 & 1.9 \\
\hline $\mathrm{pX}$ & 6 & 6 & 2.3 & 6 & 8 & 2.1 \\
\hline
\end{tabular}

Figure 5a. There is no barrier to the reorientation of the benzene molecule in the center of the cage, as the molecule is small enough that it can pass through the void without a steric clash. Therefore, the only diffusion barrier, which can be expected to be rate-determining, is for diffusion out of the cage window. Figure $4 \mathrm{~b}$ confirms this interpretation as it shows that the maxima in the energy profile appear when the benzene is in the window itself. A representative configuration adopted by the benzene molecule when it is in the window is shown in the central panel of Figure 5a, and the rightmost panel of Figure 5a shows a typical configuration for this system when the benzene is fully outside of the cage molecule. The window diffusion barrier for benzene was found to be $35 \mathrm{~kJ} \mathrm{~mol}^{-1}$ and is comparable to those calculated in the past for large guest molecules, such as $\mathrm{SF}_{6}$, in CC3. ${ }^{25}$

By studying the diffusion of toluene (Figure 4c, top center), the effect that adding a single methyl group to the benzene ring has upon the diffusion of the guest in CC3 was examined. Similarly to benzene, there is no discernible barrier to the reorientation of toluene in the CC3 void, but toluene has a higher energy when it is close to the cage center of mass. This increase indicates that toluene is a worse fit in the cage void than benzene. Having said that, the window barrier for toluene, which is once again the rate-determining step for the diffusion, is $25 \mathrm{~kJ} \mathrm{~mol}^{-1}$, which is $10 \mathrm{~kJ} \mathrm{~mol}^{-1}$ smaller than the barrier for benzene. The window diffusion mechanism for toluene (Figure 5b) demonstrates that the molecule's methyl 
(a)

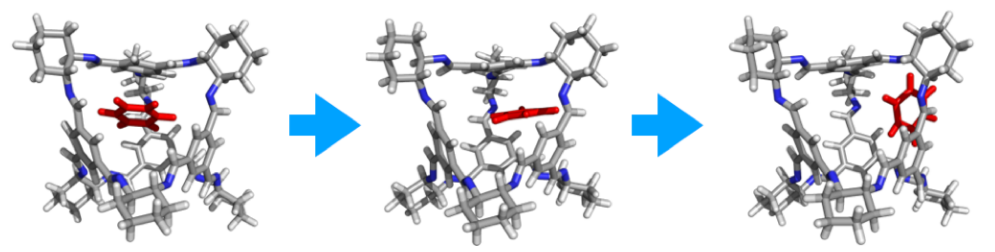

(b)

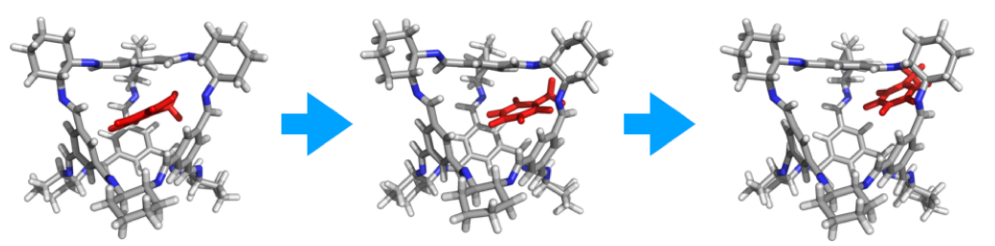

(c)

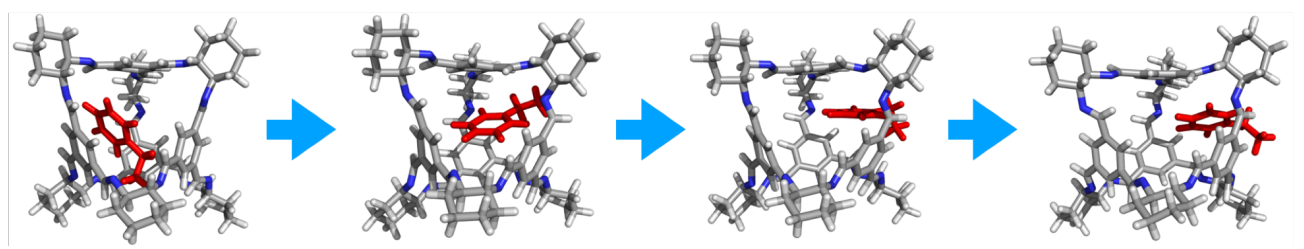

(d)

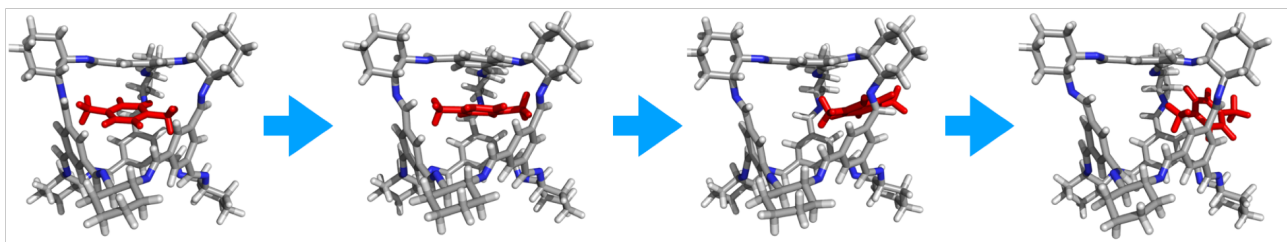

(e)

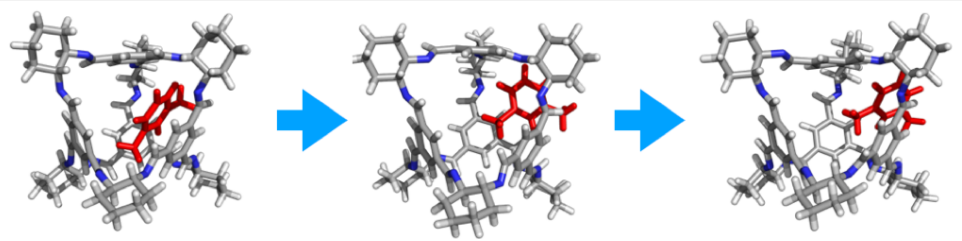

(f)

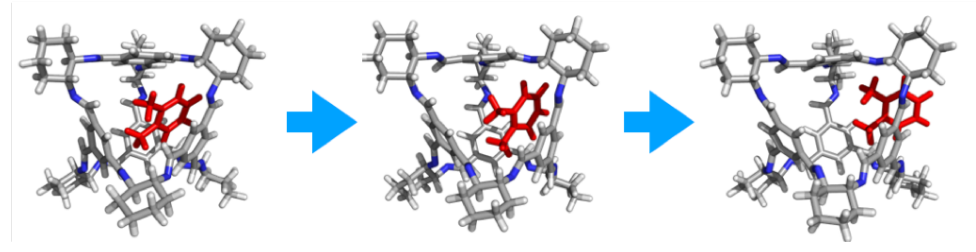

Figure 5: Snapshots of trajectories in which a) benzene, b) toluene, c) ethylbenzene, d) $\mathrm{pX}$, e) $\mathrm{mX}$, and f) oX are exiting the porous organic cage $\mathbf{C C} 3$ (gray $=\mathrm{C}$, white $=\mathrm{H}$, blue $=\mathrm{N}$; the guest molecule is entirely colored in red). In a), b), e), and f), the three images correspond to, from left to right, a trajectory frame where the guest was fully in the cage, a frame where the guest was in the cage window, and a frame where the guest was entirely outside the cage. In c) and d), an additional frame within the cage is included, so a reorientation within the cage can be seen. 
group leads it out of the cage. When packed in the solid state, the methyl group would also lead the toluene molecule into a neighboring cage through that cage's window. Because the methyl group is narrower than the aromatic ring, a preference for passing through the window methyl-first would imply a two-step cooperative diffusion mechanism in which the methyl group enters the window first and slightly enlarges it, subsequently making it easier to accommodate the ring. This cooperative diffusion mechanism is impossible for benzene, which may explain why it has a bigger window crossing barrier.

Increasing the size of the substituent by replacing the methyl group in toluene with the ethyl group in ethylbenzene (Figure 4c, top right) brings more significant changes to the FES and the minimum energy path. The center of mass of the cage is no longer the lowest energy region of the FES; instead, low energy regions are located just inside the windows, as the longer length of ethylbenzene means that it sits with its center of mass slightly towards one of the cage windows. This can be seen in Figure 5c. In the first of the images in Figure 5c, the molecule is $\pi$-stacked with the cage wall but offset from the center of mass. In the second image, the ring of ethylbenzene is close to the center of the cage void, but the overall center of mass of ethylbenzene has moved toward the window. This behavior is consistent with the route calculated for the ethylbenzene minimum energy path (shown in the FES in Figure 4c), which avoids the cage center of mass. Furthermore, there is now an energetic barrier to the reorientation shown in the first two images of Figure $5 \mathrm{c}$, which has a magnitude of $7 \mathrm{~kJ} \mathrm{~mol}^{-1}$ (Figure 4b). However, the window barrier, which has a magnitude of $13 \mathrm{~kJ} \mathrm{~mol}^{-1}$, is still ratedetermining. The decrease in the window barrier compared to benzene and toluene is because of the substituent on the molecule, which enables a cooperative diffusion mechanism much like that observed for toluene. Figure $5 \mathrm{c}$ shows ethylbenzene passing through the window ethyl first, but in this case, the substituent effect is more significant because the diameter of the ethyl group is larger than the diameter of the methyl group.

Further analysis was undertaken to establish whether the mechanism shown in Figure 5c is the only window crossing mechanism for ethylbenzene. In theory, the benzene ring can 
leave the cage before the ethyl group, and this mechanism's crossing barrier could have a different energy. To investigate the likelihood for this mechanism, an additional metadynamics calculation was performed using an additional collective variable that measured the orientation of ethylbenzene with respect to the vector connecting the center of the ring of ethylbenzene and the cage center of mass. The methods and results for this analysis are discussed further in Section S5.

The relationship between the substituents and their FES is more complicated for the xylene isomers (Figure 4c, bottom row) than for benzene, toluene or ethylbenzene. For pX (Figure 4c, bottom left), the FES is qualitatively similar to the FES for ethylbenzene. Figure $5 \mathrm{~d}$ shows that the lowest energy sites for the guest molecule are also located just inside the window. Adding the additional methyl in a para-position compared to toluene, shifts both the reorientation and window crossing barriers to approximately $6 \mathrm{~kJ} \mathrm{~mol}^{-1}$, which means that for $\mathrm{pX}$ both barriers will determine the overall diffusion rate. Figure $5 \mathrm{~d}$ explains both the presence of the reorientation barrier and why the window barrier is smaller than for ethylbenzene, toluene or benzene. As was the case for ethylbenzene, the molecule is too long to adequately occupy the cage center of mass, which is highlighted by the energy spike around the center of mass in Figure 4c. Additionally, the first two images in Figure 5d show that the length of the $\mathrm{pX}$ molecule forces at least one of the methyl substituents into a window whenever the guest is inside the cage. Having the substituent in the window in this way results in a cooperative diffusion mechanism such as those described for toluene and ethylbenzene, but one that can occur much more frequently than the cooperative diffusion of the other two guests because the substituents of $\mathrm{pX}$ spend so much time in the window.

The FES for $\mathrm{mX}$ (Figure 4c, bottom center) was found to be qualitatively similar to those for benzene and toluene. The preferred path also passes close to the cage center of mass and, while a reorientation barrier of $2 \mathrm{~kJ} \mathrm{~mol}^{-1}$ is present, it is both approximately equal to the calculated error in the FES and dwarfed by the window crossing barrier of $43 \mathrm{~kJ} \mathrm{~mol}^{-1}$. The positions of the methyl groups on the ring in $\mathrm{mX}$ mean that the ring is able to sit against the 
cage wall with the substituents aligned with the windows, but not protruding out of them as was seen for $\mathrm{pX}$. It is therefore much less unfavourable to have $\mathrm{mX}$ at the center of the cage void than to have ethylbenzene and pX. Figure 5e shows that the substituents again enable a cooperative diffusion mechanism, with one methyl group being the first part of the molecule to pass through the window and the other methyl group being the last. Furthermore, the molecule rotates during the crossing such that the broadest part of the molecule is in the window for the minimum length of time. A configuration in which $\mathrm{mX}$ is in the window is shown in the second image of Figure 5e, with the cage deforming slightly to allow this to happen. It is this brief motion that is responsible for the sharp maximum seen in the energy profile in Figure 4b, which is the most massive energy barrier of any of the molecules in this study.

oX (Figure 4c, bottom right) also behaves similarly to benzene and toluene. The preferred path for this molecule passes close to the cage's center of mass, has a small reorientation barrier (again approximately equal to the FES's error), and has a rate-determining window diffusion barrier of $35 \mathrm{~kJ} \mathrm{~mol}^{-1}$, which is very similar to that of benzene. The mechanism by which oX leaves the cage is shown in Figure $5 \mathrm{f}$. The only substituent effect, in this case, is that oX prefers to leave via the unsubstituted side first, as the two methyl groups are too close together for either to enable a cooperative diffusion mechanism.

The results summarized above indicate that diffusion through the CC3 window will be the main bottleneck for diffusion through the extended network and, therefore, for a kinetic separation in CC3. For the four C8 aromatics (Figure 1), the order of window barrier size, which can be predicted to be inversely proportional to diffusivity in CC3, was $\mathrm{mX}\left(42 \mathrm{~kJ} \mathrm{~mol}^{-1}\right)>\mathrm{oX}\left(35 \mathrm{~kJ} \mathrm{~mol}^{-1}\right)>$ ethylbenzene $\left(13 \mathrm{~kJ} \mathrm{~mol}^{-1}\right)>\mathrm{pX}\left(6 \mathrm{~kJ} \mathrm{~mol}^{-1}\right)$. The difference between the diffusion barriers for $\mathrm{pX}$ compared to $\mathrm{mX}\left(36 \mathrm{~kJ} \mathrm{~mol}^{-1}\right)$ and oX $\left(29 \mathrm{~kJ} \mathrm{~mol}^{-1}\right)$ suggests that CC3 would be highly selective for separating $\mathrm{pX}$ from $\mathrm{mX}$ and oX via a membrane or chromatographic separation. The difference in the diffusion barriers is much larger than those calculated for the experimentally proven separation of 
Xe and Kr using the same method $\left(3 \mathrm{~kJ} \mathrm{~mol}^{-1}\right) .{ }^{47}$ Even the difference between the barriers for $\mathrm{pX}$ and ethylbenzene $\left(7 \mathrm{~kJ} \mathrm{~mol}^{-1}\right)$ was greater than the value for the two noble gases, which indicates that CC3 could potentially also separate ethylbenzene from a mixture of $\mathrm{C} 8$ aromatics, albeit with a lower selectivity than the separation of $\mathrm{mX}$ and oX from the mixture. As noted above, this selectivity is a result of the predicted diffusion mechanisms, which are cooperative diffusion mechanisms that are enabled by the guests' substituents. Membrane separations governed by configurational effects have long been known. Singh and Koros found that rotational effects were responsible for the ability of zeolite $4 \mathrm{~A}$ to separate $\mathrm{N}_{2}$ and $\mathrm{O}_{2}$; these gas molecules, like the four aromatics with a kinetic diameter of $5.8 \AA$, have a similar cross-section but lengths different enough to result in a selective separation. ${ }^{60}$ Additionally, in studies of gas separations in CMS membranes, molecular-sieving effects have proven able to govern sorption selectivity as well as diffusion selectivity, ${ }^{61}$ which could indicate that the kinetic selectivity of the C8 aromatics may result in a significant difference in permeability in a real membrane.

\section{Diffusion Free Energy Barriers in CC13}

In order to establish whether similar imine cages showed the same behavior as CC3 and to investigate the impact that small modifications to the cage structure have on guest diffusion barriers, equivalent metadynamics simulations were carried out using the porous organic cage CC13 (Figure 2a). The FESs for CC13 (Figure 6a) and energy profiles of the minimum energy pathways (Figure 6b) broadly show the same features found for CC3 (Figure 4). However, they are more noisy and are less symmetrical than the CC3 plots. The reduction in symmetry is mainly because of the asymmetry of the CC13 molecule itself, which comes about because each linker has two possible orientations (see Section S1 for further details). Therefore, unlike in CC3, the four windows of a CC13 isomer are often not equivalent, although, as shown in Figure S1, the windows behave similarly across all the possible isomers. In all of the simulations the two window barriers differed by less than $10 \%$ of the larger value. 
Some of the noise is also likely a result of the increased flexibility of the cage, which proved problematic when setting up the simulations (see section S2.1 for details of the additional constraints that were included in the simulations and which may have affected the FESs in Figure 6a). As in Figure 4c, the FES plots in Figure 6a feature approximate minimum energy paths. The exact paths that were calculated using the MEPSA code ${ }^{54}$ are shown in Figure S16b. The window and reorientation barrier sizes for CC13 are shown and compared to the equivalent values for CC3 in Table 1. 
(a)
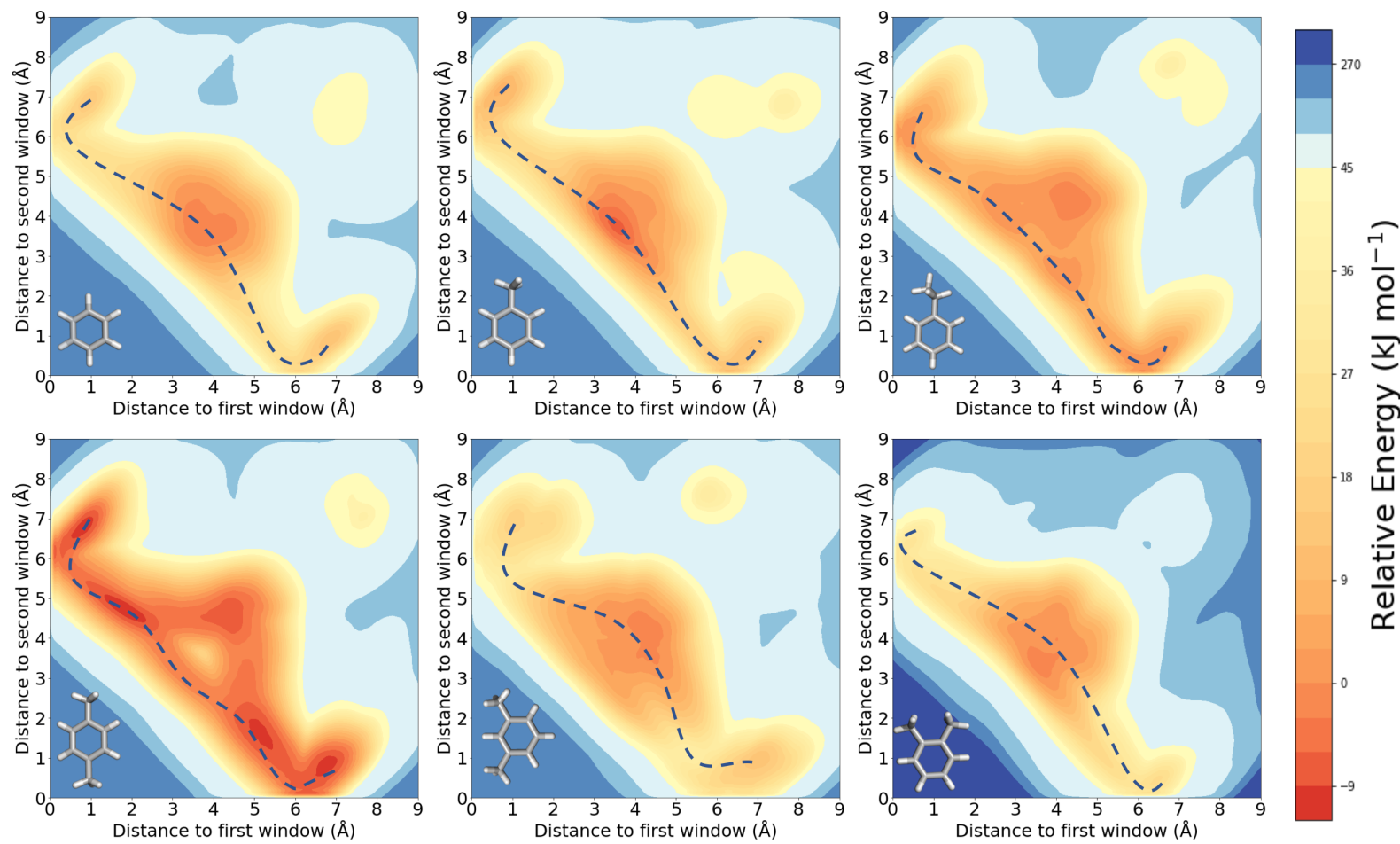

(b)

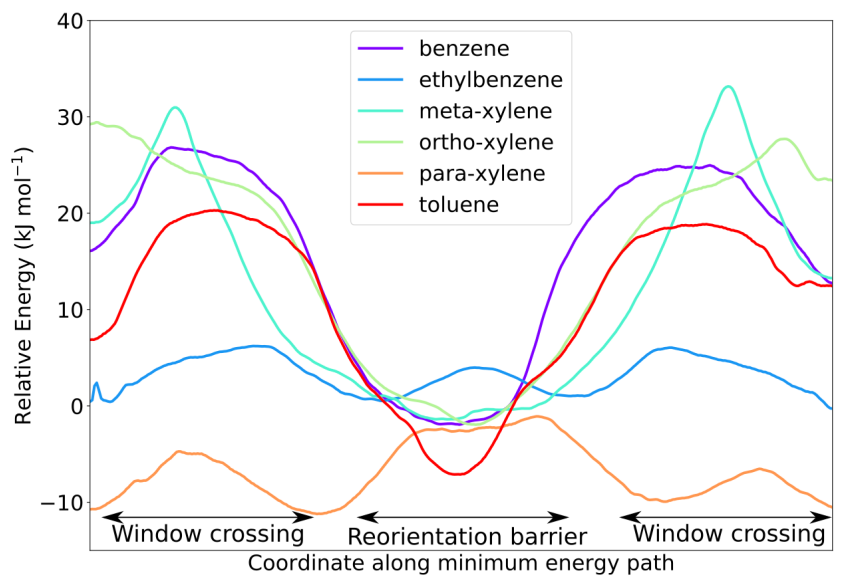

Figure 6: a) The 2D FES for each simulation with CC13, including an approximate minimum energy path (dotted line). b) The energy profile for the minimum energy path for each aromatic guest crossing through a CC13 cage.

The same trend that was observed for the CC3 window barriers was also observed for the CC13 window barriers, which indicates that the guest molecules in CC13 also diffuse via the same cooperative diffusion mechanism observed in CC3 (outlined in the snapshots in Figure 5). Four out of six guests showed smaller window barriers in CC13 compared 
to CC3, with the barriers for $\mathrm{pX}$ and toluene being equivalent. These changes in the magnitudes of the barriers ensured that the differences in the window barriers of the C8 aromatics are decreased, suggesting that CC13 will have a slightly poorer potential guest selectivity. Having said that, however, the differences between the barrier for $\mathrm{pX}$ and the barriers for $\mathrm{mX}$ and oX (28 and $25 \mathrm{~kJ} \mathrm{~mol}^{-1}$ respectively) are still more than enough to allow for an effective separation. The difference between the barriers for ethylbenzene and $\mathrm{pX}$ is $1.5 \mathrm{~kJ} \mathrm{~mol}^{-1}$, which is probably too small for CC13 to represent a viable separating agent for ethylbenzene and pX (this value is very similar to the error calculated for the FESs). Similarly to CC3, the reorientation barrier will likely have a negligible impact on diffusion rates through CC13 for all the guest molecules apart from ethylbenzene and pX; in CC13, these were the only two guests to have a measurable reorientation barrier at all. The reorientation barrier for $\mathrm{pX}$ (Figure $6 \mathrm{~b}$, bottom center) is $2 \mathrm{~kJ} \mathrm{~mol}^{-1}$ larger than the window barrier, but this difference is unlikely to affect the diffusion selectivity of the cage.

\section{Importance of Window Breathing for Small Molecule Diffusion}

In order to investigate the difference in the diffusion behavior of the aromatic molecules between the two cage systems, the structural differences of the cages were explored by calculating their PLEs with and without the C8 aromatics present. Pore breathing could explain the predicted lower selectivity of CC13, and could also further justify the observation that the C8 aromatics diffuse through the cage windows via cooperative diffusion mechanisms. The PLEs of both cages are shown in Figure 7. The PLE of the empty CC3 cage has a mode of $3.74 \AA$, which is smaller than the mode of $3.88 \AA$ that is observed for CC13. Additionally, the CC13 PLE includes larger window sizes than the window of CC3, especially when the guest molecules are in the cage windows. For example, the PLE of empty CC13 features window diameters that are larger than $5 \AA$, which are larger than any of the diameters seen in the empty CC3 PLE. With the guest molecules in the window, the same effect is seen at even larger window diameters. Diameters that are larger than the kinetic diameter of 
ethylbenzene and $\mathrm{pX}$ are thus more common in the PLEs for CC13 when compared to the equivalent PLEs for CC3. This result implies that the windows of CC13 are better at accommodating large aromatic molecules such as xylene isomers.

(a)

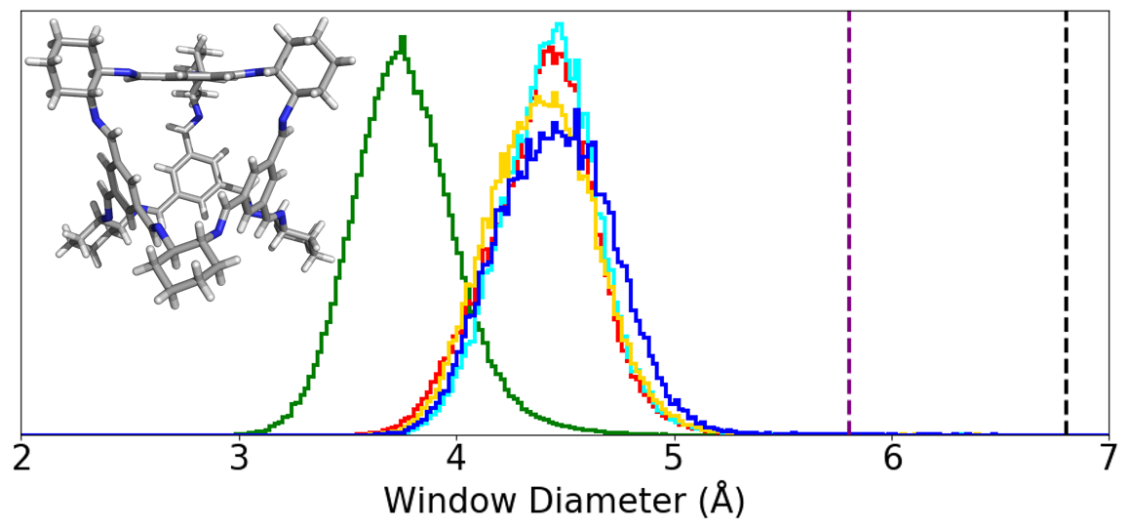

(b)

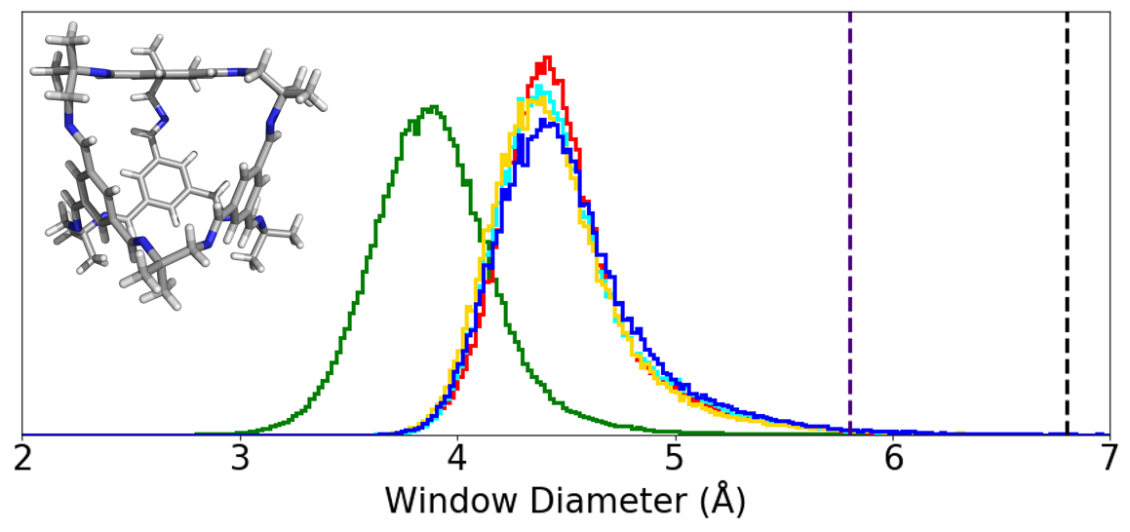

Figure 7: Pore limiting envelopes for (a) CC3 and (b) CC13 for empty cages (green) and cages with guests in the window: ethylbenzene (red), $\mathrm{pX}$ (cyan), $\mathrm{mX}$ (yellow) and oX (blue). PLEs were normalized to a probability density using a matplotlib internal function. Dotted lines represent the kinetic diameters of ethylbenzene and $\mathrm{pX}$ (purple), and $\mathrm{mX}$ and $\mathrm{oX}$ (black). Single molecules of the two cages are shown in the upper left corner of each plot, with their window facing out of the plane of the page.

The PLEs with guests present show larger window diameters than the PLEs for the empty cages, which provides further evidence for the cooperative diffusivity mechanism discussed in this work. A similar change in window diameter has also been observed during the cooperative diffusion of $\mathrm{SF}_{6} .{ }^{25}$ The larger window diameters were seen in all PLEs in CC13, but especially those with guest molecules present. This result indicates that dynamic porosity 
caused by the breathing motions of the windows of CC13 is a more significant aid to the cooperative diffusion of the guests in CC13 than in CC3. This higher degree of dynamic porosity explains why the majority of the window barriers in CC13 are smaller in magnitude than their equivalents in CC3, and why this effect was most significant for $\mathrm{mX}$ and oX, the two molecules with a larger kinetic diameter. The likely explanation for the greater flexibility of CC13 is that the linker used in CC13 allows more conformational freedom for the cage than the cyclohexane vertices.

\section{Conclusions}

Metadynamics simulations were used to explore whether two porous organic cages, the widely studied CC3, and the structurally similar CC13, could be used to separate the three isomers of xylene and their structural isomer ethylbenzene. These cages have both been successfully used to fabricate amorphous membranes, ${ }^{29}$ and are thus candidates for use in membrane separations. A set of two collective variables was developed to calculate the free energetic barriers associated with $\mathrm{pX}, \mathrm{oX}, \mathrm{mX}$, ethylbenzene, benzene and toluene diffusing through a porous organic cage molecule, and several significant potential barriers to the guests' diffusion - passing through a cage window and reorienting within a cage - were investigated. These calculations showed that, with the exception of the two longest molecules, ethylbenzene and pX, the window barrier is significantly greater in magnitude than the reorientation barrier, and will likely be rate-determining for diffusion through the cage pore networks.

In both cages, the window barrier for pX diffusion was more than $25 \mathrm{~kJ} \mathrm{~mol}^{-1}$ smaller than those for $\mathrm{mX}$ and $\mathrm{oX}$, which indicates that both cages should kinetically separate $\mathrm{pX}$ from the other xylene isomers with high selectivity. In CC3, there was also a difference of $7 \mathrm{~kJ} \mathrm{~mol}^{-1}$ between the window barriers for ethylbenzene and pX, so CC3 may also be able to separate $\mathrm{pX}$ and ethylbenzene, albeit with lower selectivity than the separation of the three xylene isomers. This separation is probably not possible with CC13 as the barriers to 
the diffusion of $\mathrm{pX}$ and ethylbenzene differ by just $1.5 \mathrm{~kJ} \mathrm{~mol}^{-1}$.

The selectivity predicted for the separation of the C8 aromatics in CC3 was justified by exploring the mechanisms by which each guest crosses through the window. Ethylbenzene, $\mathrm{pX}$, and toluene all passed through the window using cooperative diffusion mechanisms aided by their substituents, which resulted in lower window barriers than benzene. The positions of the substituents on $\mathrm{mX}$ and oX prevented them from gaining the benefits of cooperative diffusion to the same extent, however, as the substituent effects were offset by the larger diameters of $\mathrm{mX}$ and oX. The lower selectivity of CC13 for the separation of the C8 aromatics was justified by comparing the cages' allowed window sizes. The CC13 window was found to be much more likely to open wide enough to accommodate large aromatic molecules.

Overall, the metadynamics simulations were able quantify the energy barriers to diffusion of C8 aromatics through two cages, thus giving a qualitative idea of their selectivity. The simulations also allowed us to discover the key factors that affect the size of these barriers and showed that the differences between the guest molecules were due to differences in the cooperative diffusion mechanisms, while the differences between the two cages could be accounted for by considering pore breathing. This knowledge could be used to develop a screening process further for existing and hypothetical porous organic cages for their ability to perform the separation of C8 isomers and for other molecular separations.

\section{Acknowledgement}

The authors thank BP for technical support and for providing funding via an EPSRC Industrial CASE award. KEJ thanks the Royal Society for a University Research Fellowship, the EPSRC (EP/M017257/1), and the ERC (Grant Agreement 758370, ERC-StG-PE5CoMMaD) for funding. We would like to thank Dr Enrico Berardo, Dr Andrew Tarzia and Dr James Pegg for valuable discussions. 


\section{Supporting Information Available}

The following files are available free of charge: Further computational details for the metadynamics simulations, including input files; plots showing the metadynamics simulations' convergence; and additional plots and analysis of the results (PDF).

This material is available free of charge via the Internet at http://pubs.acs.org/.

\section{References}

(1) Humphrey, J. L.; Keller, G. E. Separation Process Technology; McGraw-Hill: New York, 1997.

(2) Sholl, D. S.; Lively, R. P. Seven chemical separations to change the world. Nature 2016, 532, 435-437.

(3) Kuczenski, B.; Geyer, R. Material flow analysis of polyethylene terephthalate in the US, 1996-2007. Resources, Conservation and Recycling 2010, 54, 1161-1169.

(4) Cannella, W. J. In Kirk-Othmer Encyclopedia of Chemical Technology, 5th ed.; KirkOthmer,, Ed.; John Wiley \& Sons, 2007; pp 1-42.

(5) Mohameed, H.; Jdayil, B. A.; Takrouri, K. Separation of para-xylene from xylene mixture via crystallization. Chemical Engineering and Processing 2007, 46, 25-36.

(6) Yang, Y.; Bai, P.; Guo, X. Separation of xylene isomers: a review of recent advances in materials. Industrial \& Engineering Chemistry Research 2017, 56, 14725-14753.

(7) Gonçalves, J. C.; Rodrigues, A. E. Xylene isomerization in the liquid phase using largepore zeolites. Chemical Engineering \& Technology 2016, 39, 225-232.

(8) Minceva, M.; Rodrigues, A. E. Understanding and revamping of industrial scale SMB units for p-xylene separation. AIChE Journal 2007, 53, 138-149. 
(9) Lim, Y. I.; Lee, J.; Bhatia, S. K.; Lim, Y. S.; Han, C. Improvement of para-xylene SMB process performance on an industrial scale. Industrial and Engineering Chemistry Research 2010, 49, 3316-3327.

(10) Bao, Z.; Chang, G.; Xing, H.; Krishna, R.; Ren, Q.; Chen, B. Potential of microporous metal-organic frameworks for separation of hydrocarbon mixtures. Energy and Environmental Science 2016, 9, 3612-3641.

(11) Toda, J.; Corma, A.; Sastre, G. Diffusion of trimethylbenzenes and xylenes in zeolites with 12- and 10-ring channels as catalyst for toluene-trimethylbenzene transalkylation. The Journal of Physical Chemistry C 2016, 120, 16668-16680.

(12) Torres-Knoop, A.; Krishna, R.; Dubbeldam, D. Separating xylene isomers by commensurate stacking of p-xylene within channels of MAF-X8. Angewandte Chemie International Edition 2014, 53, 7774-7778.

(13) Gee, J. A.; Zhang, K.; Bhattacharyya, S.; Bentley, J.; Rungta, M.; Abichandani, J. S.; Sholl, D. S.; Nair, S. Computational identification and experimental evaluation of metalorganic frameworks for xylene enrichment. Journal of Physical Chemistry C 2016, 120, $12075-12082$.

(14) Lennox, M. J.; Düren, T. Understanding the kinetic and thermodynamic origins of xylene separation in UiO-66(Zr) via molecular simulation. The Journal of Physical Chemistry C 2016, 120, 18651-18658.

(15) Lai, Z.; Bonilla, G.; Diaz, I.; Nery, J. G.; Sujaoti, K.; Amat, M. A.; Kokkoli, E.; Terasaki, O.; Thompson, R. W.; Tsapatsis, M. et al. Microstructural optimization of a zeolite membrane for organic vapor separation. Science 2003, 300, 456-460.

(16) Choi, J.; Jeong, H.-K.; Snyder, M. A.; Stoeger, J. A.; Masel, R. I.; Tsapatsis, M. Grain Boundary Defect Elimination in a Zeolite Membrane by Rapid Thermal Processing. Science 2009, 325, 590-593. 
(17) Varoon, K.; Zhang, X.; Elyassi, B.; Brewer, D. D.; Gettel, M.; Kumar, S.; Lee, J. A.; Maheshwari, S.; Mittal, A.; Sung, C. Y. et al. Dispersible exfoliated zeolite nanosheets and their application as a selective membrane. Science 2011, 334, 72-75.

(18) Banihashemi, F.; Meng, L.; Babaluo, A. A.; Lin, Y. S. Xylene vapor permeation in MFI zeolite membranes made by templated and template-free secondary growth of randomly oriented seeds: effects of xylene activity and microstructure. Industrial and Engineering Chemistry Research 2018, 5\%, 16059-16068.

(19) Bilaus, R. S. Membrane materials and technology for xylene isomers separation and isomerization via pervaporation. MSc Dissertation, King Abdullah University of Science and Technology, 2014.

(20) Koh, D.-Y.; McCool, B. A.; Deckman, H. W.; Lively, R. P. Reverse osmosis molecular differentiation of organic liquids using carbon molecular sieve membranes. Science 2016, 353 .

(21) Evans, J. D.; Sumby, C. J.; Doonan, C. J. Synthesis and applications of porous organic cages. Chemistry Letters 2015, 44, 582-588.

(22) Hasell, T.; Cooper, A. I. Porous organic cages: soluble, modular and molecular pores. Nature Reviews Materials 2016, 1, 16053.

(23) Beuerle, F.; Gole, B. Covalent organic frameworks and cage compounds: design and applications of polymeric and discrete organic scaffolds. Angewandte Chemie - International Edition 2018, 57, 4850-4878.

(24) Liu, M.; Little, M. A.; Jelfs, K. E.; Jones, J. T. A.; Schmidtmann, M.; Chong, S. Y.; Hasell, T.; Cooper, A. I. Acid- and base-stable porous organic cages: shape persistence and $\mathrm{pH}$ stability via post-synthetic "tying" of a flexible amine cage. Journal of the American Chemical Society 2014, 136, 7583-7586. 
(25) Hasell, T.; Miklitz, M.; Stephenson, A.; Little, M. A.; Chong, S. Y.; Clowes, R.; Chen, L.; Holden, D.; Tribello, G. A.; Jelfs, K. E. et al. Porous organic cages for sulfur hexafluoride separation. Journal of the American Chemical Society 2016, 138, $1653-1659$.

(26) Chen, L.; Reiss, P. S.; Chong, S. Y.; Holden, D.; Jelfs, K. E.; Hasell, T.; Little, M. A.; Kewley, A.; Briggs, M. E.; Stephenson, A. et al. Separation of rare gases and chiral molecules by selective binding in porous organic cages. Nature Materials 2014, 13, 954-960.

(27) Mitra, T.; Jelfs, K. E.; Schmidtmann, M.; Ahmed, A.; Chong, S. Y.; Adams, D. J.; Cooper, A. I. Molecular shape sorting using molecular organic cages. Nature Chemistry 2013, 5, 276-281.

(28) Slater, A. G.; Cooper, A. I. Function-led design of new porous materials. Science 2015, 348 , aaa 8075 .

(29) Song, Q.; Jiang, S.; Hasell, T.; Liu, M.; Sun, S.; Cheetham, A. K.; Sivaniah, E.; Cooper, A. I. Porous organic cage thin films and molecular-sieving membranes. Advanced Materials 2016, 28, 2629-2637.

(30) Jiang, S.; Song, Q.; Massey, A.; Chong, S. Y.; Chen, L.; Sun, S.; Hasell, T.; Raval, R.; Sivaniah, E.; Cheetham, A. K. et al. Oriented two-dimensional porous organic cage crystals. Angewandte Chemie - International Edition 2017, 56, 9391-9395.

(31) Skowronek, P.; Gawronski, J. Chiral iminospherand of a tetrahedral symmetry spontaneously assembled in a [6+4] cyclocondensation. Organic Letters 2008, 10, 4755-4758.

(32) Tozawa, T.; Jones, J. T. A.; Swamy, S. I.; Jiang, S.; Adams, D. J.; Shakespeare, S.; Clowes, R.; Bradshaw, D.; Hasell, T.; Chong, S. Y. et al. Porous organic cages. Nature Materials 2009, 8, 973-978. 
(33) Hasell, T.; Culshaw, J. L.; Chong, S. Y.; Schmidtmann, M.; Little, M. A.; Jelfs, K. E.; Pyzer-Knapp, E. O.; Shepherd, H.; Adams, D. J.; Day, G. M. et al. Controlling the crystallization of porous organic cages: molecular analogs of isoreticular frameworks using shape-specific directing solvents. Journal of the American Chemical Society 2014, $136,1438-1448$.

(34) Jiang, S.; Jelfs, K. E.; Holden, D.; Hasell, T.; Chong, S. Y.; Haranczyk, M.; Trewin, A.; Cooper, A. I. Molecular dynamics simulations of gas selectivity in amorphous porous molecular solids. Journal of the American Chemical Society 2013, 135, 17818-17830.

(35) Holden, D.; Jelfs, K. E.; Cooper, A. I.; Trewin, A.; Willock, D. J. Bespoke force field for simulating the molecular dynamics of porous organic cages. Journal of Physical Chemistry $C$ 2012, 116, 16639-16651.

(36) Holden, D.; Jelfs, K. E.; Trewin, A.; Willock, D. J.; Haranczyk, M.; Cooper, A. I. Gas diffusion in a porous organic cage: analysis of dynamic pore connectivity using molecular dynamics simulations. The Journal of Physical Chemistry C 2014, 118, 1273412743.

(37) Manurung, R.; Holden, D.; Miklitz, M.; Chen, L.; Hasell, T.; Chong, S. Y.; Haranczyk, M.; Cooper, A. I.; Jelfs, K. E. Tunable porosity through cooperative diffusion in a multicomponent porous molecular crystal. The Journal of Physical Chemistry C 2015, 119, 22577-22586.

(38) Holden, D.; Chong, S. Y.; Chen, L.; Jelfs, K. E.; Hasell, T.; Cooper, A. I. Understanding static, dynamic and cooperative porosity in Molecular Materials. Chemical Science 2016, 7, 4875-4879.

(39) Barbour, L. J. Crystal porosity and the burden of proof. Chemical Communications 2006, 1163-1168. 
(40) Verploegh, R. J.; Nair, S.; Sholl, D. S. Temperature and loading-dependent diffusion of light hydrocarbons in ZIF-8 as predicted through fully flexible molecular simulations. Journal of the American Chemical Society 2015, 137, 15760-15771.

(41) Dubbeldam, D.; Beerdsen, E.; Vlugt, T. J. H.; Smit, B. Molecular simulation of loadingdependent diffusion in nanoporous materials using extended dynamically corrected transition state theory. Journal of Chemical Physics 2005, 122.

(42) Boulfelfel, S. E.; Ravikovitch, P. I.; Sholl, D. S. Modeling diffusion of linear hydrocarbons in silica zeolite LTA using transition path sampling. Journal of Physical Chemistry C 2015, 119, 15643-15653.

(43) Camp, J. S.; Sholl, D. S. Transition state theory methods to measure diffusion in flexible nanoporous materials: application to a porous organic cage crystal. The Journal of Physical Chemistry C 2016, 120, 1110-1120.

(44) Valsson, O.; Tiwary, P.; Parrinello, M. Enhancing important fluctuations: rare events and metadynamics from a conceptual viewpoint. Annual Review of Physical Chemistry 2016, 67, 159-184.

(45) Roux, B. The calculation of the potential of mean force using computer simulations. Computer Physics Communications 1995, 91, 275-282.

(46) Laio, A.; Parrinello, M. Escaping free-energy minima. Proceedings of the National Academy of Sciences 2002, 99, 12562-12566.

(47) Miklitz, M.; Jiang, S.; Clowes, R.; Briggs, M. E.; Cooper, A. I.; Jelfs, K. E. Computational screening of porous organic molecules for xenon/krypton separation. The Journal of Physical Chemistry C 2017, 121, 15211-15222.

(48) Smith, W.; Yong, C. W.; Rodger, P. M. DL_POLY: Application to molecular simulation. Molecular Simulation 2002, 28, 37-41. 
(49) Yong, C. W. In CSE Frontier; Blake, R., Ed.; STFC Computational Science and Engineering: Daresbury Laboratory, 2010; pp 38-40.

(50) Yong, C. W. Descriptions and implementations of DL_F notation: a natural chemical expression system of atom types for molecular simulations. Journal of Chemical Information and Modeling 2016, 56, 1405-1409.

(51) Banks, J. L.; Beard, H. S.; Cao, Y.; Cho, A. E.; Damm, W.; Farid, R.; Felts, A. K.; Halgren, T. A.; Mainz, D. T.; Maple, J. R. et al. Integrated modeling program, applied chemical theory (IMPACT). Journal of Computational Chemistry 2005, 26, 1752-1780.

(52) Jelfs, K. E.; Eden, E. G. B.; Culshaw, J. L.; Shakespeare, S.; Pyzer-Knapp, E. O.; Thompson, H. P. G.; Bacsa, J.; Day, G. M.; Adams, D. J.; Cooper, A. I. In silico design of supramolecules from their precursors: odd-even effects in cage-forming reactions. Journal of the American Chemical Society 2013, 135, 9307-10.

(53) Tribello, G. A.; Bonomi, M.; Branduardi, D.; Camilloni, C.; Bussi, G. PLUMED 2 : new feathers for an old bird. Computer Physics Communications 2014, 185, 604-613.

(54) Marcos-Alcalde, I.; Setoain, J.; Mendieta-Moreno, J. I.; Mendieta, J.; GómezPuertas, P. MEPSA: minimum energy pathway analysis for energy landscapes. Bioinformatics 2015, 31, 3853-3855.

(55) Tiwary, P.; Parrinello, M. A time-independent free energy estimator for metadynamics. Journal of Physical Chemistry B 2015, 119, 736-742.

(56) Gimondi, I.; Tribello, G. A.; Salvalaglio, M. Building maps in collective variable space. Journal of Chemical Physics 2018, 149, 104104.

(57) Bussi, G.; Tribello, G. A. Analyzing and biasing simulations with PLUMED. 2018; https://arxiv.org/pdf/1812.08213.pdf. 
(58) Humphrey, W.; Dalke, A.; Schulten, K. VMD : visual molecular dynamics. Journal of Molecular Graphics 1996, 14, 33-38.

(59) Miklitz, M.; Jelfs, K. E. pywindow: automated structural analysis of molecular pores. Journal of Chemical Information and Modeling 2018, 58, 2387-2391.

(60) Singh, A.; Koros, W. J. Significance of entropic selectivity for advanced gas separation membranes. Industrial \&3 Engineering Chemistry Research 1996, 35, 1231-1234.

(61) Zhang, C.; Koros, W. J. Ultraselective carbon molecular sieve membranes with tailored synergistic sorption selective properties. Advanced Materials 2017, 29, 1-6. 
Graphical TOC Entry

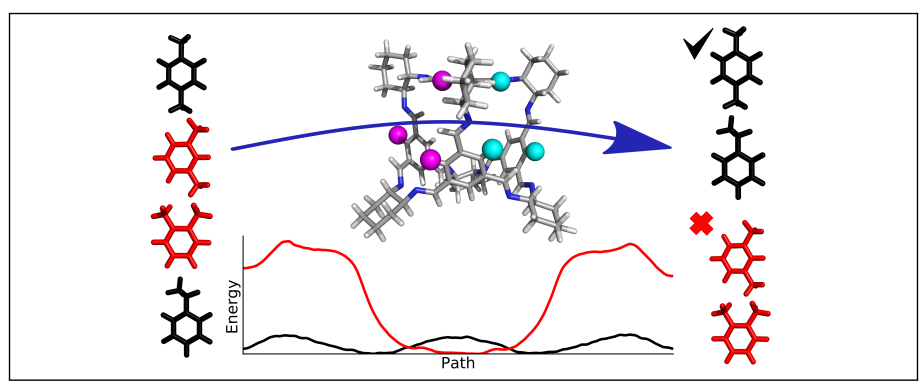

